Unilever, Bedford, UK; ${ }^{4}$ Med City Research Laboratory, University of Turku, Turku, Finland

Introduction NAFLD is characterised by steatosis, chronic inflammation and fibrosis. The underlying mechanisms include insulin resistance, increased free fatty acid flux from de-novo lipogenesis and decreased lipid oxidation. Vascular Adhesion Protein-1 (VAP -1 ), is an adhesion molecule with semicarbazide- sensitive amine oxidase activity (SSAO), which is also expressed as a soluble protein in serum (sVAP-1) and elevated in inflammatory liver diseases such as NAFLD. VAP-1 has been shown to modulate glucose and lipid uptake in muscle and adipose tissue and thus we investigated whether it may contribute to glucose and lipid homeostasis in human liver tissue.

Methods We have used precision cut liver slices (PCLS) from normal and diseased human liver specimens and cultured human sinusoidal endothelium (HSEC) and hepatocyte cells in combination with VAP-1 substrates (200 $\mu \mathrm{M}$ methylamine or benzylamine) and inhibitors ( $400 \mu \mathrm{M}$ bromoethylamine) to perform standard ex vivo radiolabelled glucose uptake and fatty acid uptake assays using oil red $O$ quantification following exposure of cells to Oleic and Palmitic acid (PA). Immunohistochemical staining and qPCR were performed using standard techniques and for confirmatory experiments HSEC were transfected with enzymatically active/inactive VAP1.

Results OPCR confirmed upregulation of VAP-1 mRNA $(\mathrm{DDCT}=1.144 \mathrm{p}=0.03)$ in NASH vs normal liver and also changes in FABP1, -4, -5, FATP3, -4 ( $\mathrm{p} \leq 0.05$ for all) and GLUT-1, 2, 3, 5, 8, 9 and 12 in NAFLD compared to normal individuals. Results were confirmed using immunohistochemical staining. Exposure of human PCLS to SVAP-1 and methylamine typically resulted in a $38 \%-54 \%$ increase in PA uptake ( $\mathrm{p} \leq 0.01$ for all) and a $20 \%$ increase in hepatocyte glucose uptake in vitro which could be inhibited using bromoethylamine. Transduction of HSEC with enzymatically active VAP-1 also increased glucose uptake which was prevented in the absence of enzyme activity. Interestingly methylamine treatment of human liver resulted in decreased expression of mRNA for glucose transporters and an increase in some lipid transporters including FABP6, FATP and LRP8, and $\mathrm{H}_{2} \mathrm{O}_{2}$ produced by SSAO activity increase lipid uptake by hepatic cells.

Conclusion In conclusion, we demonstrate for the first time global alterations in cellular expression of glucose and lipid transporter proteins in human NAFLD. We confirm that VAP-1 is elevated in disease and that SSAO activity of VAP-1 results in enhanced hepatic lipid and glucose uptake and changes in transporter expression. Thus we propose that bioactive metabolites of SSAO activity contribute to the metabolic derangement evident in fatty liver disease.

Competing interests None declared

\section{Nutrition screening}

\section{PMO-034 COMPLIANCE WITH THE MUST SCREENING TOOL FOR MEDICAL IN PATIENTS}

doi:10.1136/gutjnl-2012-302514b.34

J Middleton, ${ }^{*}$ H Farnsworth, G Singh. Gastroenterology, Bassetlaw Hospital, Worksop, UK

Introduction The National Institute for Health and Clinical Excellence (NICE) recommends that screening tools such as the five step MUST (Malnutrition universal screening tool) be used on patients admitted to hospital and weekly thereafter. ${ }^{1}$ Early identification of nutritionally deficient patients is vital so that nutritional intervention can be targeted.

Methods All patients $(n=80)$ on four general medical wards were reviewed and followed up for 4 weeks or until discharged. Medical and nursing notes were assessed to see if MUST scoring was used and if the appropriate intervention was carried out based on the MUST score. The trust policy is that $100 \%$ compliance should be achieved in the use of MUST for inpatients.

Results The Abstract PMO-034 table 1 shows that compliance with the nutritional screening tool is below the expected standard of $100 \%$ and slowly reduces over the first 4 weeks from admission. There were 14 instances of MUST score 1 but this resulted in only $36 \%$ of patients being given a high energy diet and $57 \%$ of patients being placed on a food diary. There were 28 instances of MUST score $2-3$, but this led to only $46 \%$ of patients being placed on a high energy diet and $68 \%$ of patients being placed on a food chart. There were six instances of MUST score 4-6, however this led to only $50 \%$ of patients on a high energy diet, $67 \%$ of patients being placed on a food chart although $100 \%$ of these patients were referred to a dietician.

\section{Abstract PM0-034 Table 1}

\begin{tabular}{lllll}
\hline & Week 1 & Week 2 & Week 3 & Week 4 \\
\hline Height recorded & $95 \%$ & & & \\
Weight recorded & $94 \%$ & $85 \%$ & $85 \%$ & $83 \%$ \\
BMI recorded & $88 \%$ & $89 \%$ & $82 \%$ & $82 \%$ \\
Acute disease score recorded & $82 \%$ & $83 \%$ & $77 \%$ & $81 \%$ \\
MUST score recorded & $87 \%$ & $89 \%$ & $85 \%$ & $84 \%$ \\
\hline
\end{tabular}

Conclusion The adherence to nutritional scoring in medical patients is high but below the expected $100 \%$. As time goes by the adherence to weekly screening drops slowly. Although there is a relatively high adherence to working out the MUST score, the final step in the screening tool which concentrates on nutritional intervention is disappointingly low. Continued education for both medical and nursing staff is needed so that targeted nutritional intervention can be delivered more effectively.

Competing interests None declared.

\section{REFERENCE}

1. NICE. Clinical Guidance 32. Nutrition Support in Adults. 2006

\section{PMO-035 WEIGH OFF THE MARK: AN AUDIT ON THE ACCURACY OF SCALES IN AN ACUTE HOSPITAL}

doi:10.1136/gutjnl-2012-302514b.35

K Angel,* L Silva. Department of Nutrition and Dietetics, Conquest Hospital, St Leonards-on Sea, UK

Introduction Weight is a vital component of a patient's medical information which is used in a number of assessments such as the Malnutrition Universal Screening Tool (MUST), calculating drug dosages and monitoring medical treatments. The purpose of this audit was to determine the accuracy of hospital weighing scales in accordance with the UK Weighing Federation, and investigate the impact of inaccurate weights on medical assessments.

Methods Newly calibrated scales were used to obtain a reference weight for four subjects with a range of weights: subject A $31.8 \mathrm{~kg}$, subject B $54.6 \mathrm{~kg}$, subject C $60.8 \mathrm{~kg}$ and subject D $106.7 \mathrm{~kg}$. The subjects were then weighed on all functioning hoist, chair and standing (class 3) weighing scales within the Conquest Hospital, a medium size district general hospital. The collected weights were compared to the reference weight, and then the differences compared to error allowance specified by the UK Weighing Federation for Class III scales.

Results A total of 33 scales were included in the study including one set of standing scales which produced an excessive inaccuracy. Good 
Abstract PM0-035 Table 1

\begin{tabular}{|c|c|c|c|c|c|c|}
\hline \multirow[b]{2}{*}{$\begin{array}{l}\text { Reference } \\
\text { weight (kg) }\end{array}$} & \multicolumn{2}{|l|}{ Chair scales } & \multicolumn{2}{|l|}{ Standing scales } & \multicolumn{2}{|l|}{ Hoist scales } \\
\hline & $\begin{array}{l}\text { Range of weight }(\mathrm{kg}) \\
\text { and SD }\end{array}$ & $\begin{array}{l}\text { Number within (UKWF) } \\
\text { standards (\%) }\end{array}$ & $\begin{array}{l}\text { Range of weight }(\mathrm{kg}) \\
\text { and SD }\end{array}$ & $\begin{array}{l}\text { Number within } \\
\text { (UKWF) Standards }\end{array}$ & $\begin{array}{l}\text { Range of weight } \\
\text { (kg) and SD }\end{array}$ & $\begin{array}{l}\text { Number within } \\
\text { (UKWF) standards (\%) }\end{array}$ \\
\hline 31.8 & $31.3-31.60 .13$ (SD) & $0 / 17(0 \%)$ & 15.75-31.6 5.27 (SD) & $0 / 17(0 \%)$ & 28.7-32.2 2.38 (SD) & $1 / 9(11 \%)$ \\
\hline 54.6 & & & & & 51.8-60 1.93 (SD) & $1 / 9(14 \%)$ \\
\hline 60.8 & $60.2-61.60 .25$ (SD) & $14 / 17(82 \%)$ & $30.5-61.510 .12$ (SD) & $6 / 9(67 \%)$ & & \\
\hline 106.7 & $105.6-106.70 .26$ (SD) & $9 / 17(53 \%)$ & 53.5-106.7 17.67 (SD) & $4 / 9(44 \%)$ & $103.6-107.42 .16$ (SD) & $1 / 7(14 \%)$ \\
\hline
\end{tabular}

results were obtained with the average weight subject $(60.8 \mathrm{~kg})$ with the chair and standing scales weighing within UK Weighing Federation error allowance by $82 \%$ and $67 \%$, respectively. Accuracy decreased as the weight increased as can be seen in the Abstract PMO-035 table 1. Results for subject A $(31.8 \mathrm{~kg})$ did not meet the UK Weighing Federation standards. However, at least $78 \%$ of the results from the chair and standing scales weighed within $500 \mathrm{~g}$ of all the subjects' reference weights. The largest error on the hoist scales for Subject B $(54.6 \mathrm{~kg})$ was $+5.4 \mathrm{~kg}$ increasing body mass index (BMI) by $2.0 \mathrm{~kg} / \mathrm{m}^{2}$ (height-1.64 m). A patient of similar height with a BMI of $18 \mathrm{~kg} / \mathrm{m}^{2}$ would calculate as a BMI of $20 \mathrm{~kg} / \mathrm{m}^{2}$. The MUST score would be 0 instead of 2 , which triggers a referral for dietetic assessment according to Trust policy.

Conclusion This scales audit identified that both chair and standing scales were the most accurate over the range measure $60 \mathrm{~kg}$ and $105 \mathrm{~kg}$. The hoist scales were the most inaccurate. The accuracy of all the scales decreased with the heaviest subject. With increasing levels of obesity this audit highlighted the need to calibrate scales more often and to re-audit with a wider weight range of up to $200 \mathrm{~kg}$.

Competing interests None declared.

\section{PMO-036 MALNUTRITION IN HOSPITALISED PATIENTS: DO WE ADDRESS THE PROBLEM?}

doi:10.1136/gutjnl-2012-302514b.36

M Shah, ${ }^{*}$ M Faimali. Elderly Care, Lincoln County Hospital, Lincoln, UK

Introduction Disease burden and hospital treatments are just some of the factors that contribute to the malnutrition found in hospitalised patients. Its prevalence has been documented over the years, with many studies linking its presence to a number of poorer outcomes including increased hospital stay, morbidity and mortality. The purpose of this audit was to examine whether the malnutrition universal screening tool (MUST) was used correctly and protocols followed for addressing malnutrition.

Methods We analysed the notes for all inpatients on the three care of the elderly wards at Lincoln County hospital during a 2-week period in June 2010 ( $n=90)$. Each patient's notes were evaluated to assess whether the MUST was used and if so appropriately. The audit recorded the frequency of MUST score recording and referral to dietetic/medical personnel when appropriate.

Results There were 90 patients included in this audit, 27 males and 63 females. The mean age was 79.5 years (50-99). 43 patients did not have a MUST score recorded within $24 \mathrm{~h}$ of admission and 32 did not have a weekly MUST score. Six patients required referral to dietetic/medical personnel with only two being referred. The protocol was followed correctly in 55 of the 90 patients.

Conclusion Malnutrition is a common problem within hospitalised patients. Proper nutritional care starts with the identification of at risk individuals through tools such as the MUST. In our experience the MUST tool is not used enough, delaying diagnosis with potentially worse outcomes.
Abstract PM0-036 Table 1

\begin{tabular}{lllll}
\hline $\begin{array}{l}\text { Must not } \\
\text { recorded within } \\
\text { 24 h (90) }\end{array}$ & $\begin{array}{l}\text { Must not } \\
\text { recorded } \\
\text { weekly (90) }\end{array}$ & $\begin{array}{l}\text { Referral } \\
\text { indicated (90) }\end{array}$ & $\begin{array}{l}\text { Referral } \\
\text { not made (6) }\end{array}$ & $\begin{array}{l}\text { Must } \\
\text { protocol } \\
\text { followed (90) }\end{array}$ \\
\hline 43 & 32 & 6 & 4 & 55 \\
\hline
\end{tabular}

Competing interests None declared.

\section{REFERENCES}

1. British Dietetic Association. http://www.bda.uk.com/malnutrition in hospitals. html (accessed 20 Jan 2012).

2. NICE. Nutrition Support In Adults; NICE Clinical Guideline 32. 2006:7.

3. Malnutrition Universal Screening Tool. http://scottishintensivecare.org.uk/nutrition/ docs/must.pdf (accessed 20 Jan 2012).

\section{PM0-037 ADULT NUTRITIONAL STATUS ASSESSMENT IN A HOSPITAL: CROSS SECTIONAL STUDY IN A UK HOSPITAL}

doi:10.1136/gutjnl-2012-302514b.37

${ }^{1} \mathrm{~S}$ Dingwal, ${ }^{1} \mathrm{~S}$ Shah, ${ }^{2} \mathrm{~A}$ Patel, ${ }^{3} \mathrm{~J}$ Arnold-Jellis, ${ }^{3} \mathrm{H}$ Chin, ${ }^{4} \mathrm{~S}$ Lunt, ${ }^{1} \mathrm{~S}$ R Kadri. ${ }^{1}$ Department of Gastroenterology, Luton and Dunstable Hospital, Luton, UK; ${ }^{2}$ Department of Clinical Quality, Luton and Dunstable Hospital, Luton, UK; ${ }^{3}$ Department of Clinical Nutrition, Luton and Dunstable Hospital, Luton, UK; ${ }^{4}$ Department of Dietitian, Luton and Dunstable Hospital, Luton, UK

Introduction Malnutrition in hospital can result in significant health and economic consequences. It can prolong hospital stay and can increase the risk of complications.

Objectives This cross sectional study was undertaken to assess nutrition status on acute in-patients using the Malnutrition Universal Screening Tool "MUST", and compliance of completion of nutritional and hydration documentation such as nutrition care plans, food and fluid charts.

Methods Ten patients were randomly selected from ten wards across the hospital. Wards included four Acute Medical, three Care of the Elderly and three surgical wards. All one hundred patients' health records were reviewed to identify completion of the "MUST" assessment document; nutrition care plans, and food charts. Fluid chart were also reviewed to identify compliance in completion.

Results "MUST"-Of all the patients who had a "MUST" assessment undertaken $47 \%$ (n 26/55) were found to be low risk and $52 \%$ ( $n$ 29/55) had a medium or high risk score. Two of the 10 wards had more than $50 \%$ completion. No wards had $100 \%$ completion of "MUST" assessments. Forty five percent did not have a "MUST" score. Nutrition Care Plan documentation-Eight out of ten of the wards had a nutrition care plan for all or some of their patients. $60 \%$ were completed daily. Food chart-In nine out of the 10 wards all or some patients received a food chart. $66 \%$ were partially or fully completed. Fluid charts-In nine out of ten wards all or some patients had a fluid chart and $78 \%$ were partially or fully completed. Twenty two percent did not receive a fluid chart.

Conclusion The audit found suboptimal rates of completion of "MUST" assessment and nutrition care plan documentation. Over 